\title{
Experimental Investigations on the Dynamic Behaviour of O-Type Wire-Cable Vibration Isolators
}

\author{
Hong-Xia Wang, ${ }^{1,2,3}$ Xian-Sheng Gong, ${ }^{1,2}$ Fei Pan, ${ }^{1,2}$ and Xue-Jiang Dang ${ }^{1,2}$ \\ ${ }^{1}$ The State Key Laboratory of Mechanical Transmission, Chongqing University, Chongqing 400044, China \\ ${ }^{2}$ College of Mechanical Engineering, Chongqing University, Chongqing 400044, China \\ ${ }^{3}$ Hubei Automotive Industries Institute, Shiyan, Hubei 442002, China \\ Correspondence should be addressed to Xian-Sheng Gong; cqxsgong@cqu.edu.cn
}

Received 16 July 2014; Accepted 6 October 2014

Academic Editor: Jeong-Hoi Koo

Copyright (C) 2015 Hong-Xia Wang et al. This is an open access article distributed under the Creative Commons Attribution License, which permits unrestricted use, distribution, and reproduction in any medium, provided the original work is properly cited.

\begin{abstract}
A series of periodic loading experiments were carried out on the O-type wire-cable vibration isolators. The isolators were loaded under shear, roll, and tension-compression loadings. When subjected to shear and roll loads, the restoring force-deformation curves generated by the isolators formed symmetric hysteresis loops. However, when the isolators were loaded with tension-compression loads, the isolator produced asymmetric hysteresis loops. It is found through the experiment that the dynamic characteristics of the isolator are determined by the loading amplitude as well as the geometric parameters of the isolator while they are almost independent of loading frequency within the testing frequency range. Based on the experimental data, the dynamic response of the isolator was modeled by a modified normalized Bouc-Wen model. The parameters of this model were identified through an identification procedure that does not involve any nonlinear iterative algorithms. Comparison between the identification results and the experimental data suggests that the identification method is effective. With the model and the identified parameters, the frequency response of an O-type wire-cable vibration isolator-mass system was evaluated. Typical nonlinear response behaviors were found when the isolator was used in tension-compression mode while the response appears to be similar to that of a linear system when the isolator was used in shear and roll mode.
\end{abstract}

\section{Introduction}

Wire-cable vibration isolators are typical nonlinear hysteretic damping devices. Owing to their good dry friction damping performance, wire-cable isolators have been widely used as key vibration absorption components for vibration isolation of industrial and defense equipment, naval vessels, aircraft engines and other sensitive equipment [1-4], and so forth. The isolator exhibits good damping performance due to rubbing and sliding friction between the wire strands. However, the dynamic characteristics of a conventional wire-cable isolator are controlled by several coupled parameters such as the diameter of the wire ropes, the number of strands, the cable length, the cable twist, or lay angle [5]; therefore, it is difficult to design such an isolator. Moreover, if a conventional wire-cable isolator is damaged, there is no way of fixing it but to replace it. To cope with the problems, a new Otype wire-cable vibration isolator (see Figure 1) is designed.
The O-type isolator uses independent wire rope loops as elastic-damping components. The wire rope loops can be made of complex wire ropes or simple strands depending on the usage of the isolator. In most cases, the wire ropes used are the wire rope structure with independent wire strand core (IWSC). The wire loops are held between metal retainers. Both the stiffness and the damping of the O-type wire-cable vibration isolator can be adjusted by varying the wire rope diameter, number of wire rope loops, horizontal angle of wire rope loops, and coil diameter. Compared with the conventional stranded wire helical wire-cable vibration isolator, the O-type isolator has some particular advantages, such as longer fatigue life, better installation and maintenance ease, better reliability, and better vibration absorption ability.

Full understanding of the impact of the geometric parameters on the dynamic characteristic of the isolator is of great importance for designing a wire-cable vibration isolator for engineering applications. Little research has been 


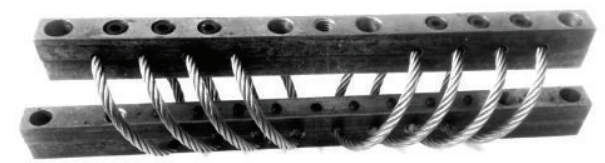

FIGURE 1: O-type wire-cable vibration isolator.

devoted to the subject. Wang et al. [6] presented a two-state model to describe the static response of the stranded wire helical springs, which are also wire rope made components, analytically and discussed the load-strain relationship of the springs with different geometric parameters. Gong and Tang [7] presented a comprehensive model for nonlinear hysteretic systems. Gerges [8] and Gerges and Vickery [9] presented a semianalytical model for wire rope vibration isolator under tension-compression loading and proposed two mathematical models for describing the effective stiffness and the equivalent viscous damping ratio of conventional wire rope springs. $\mathrm{Ni}$ et al. $[5,10]$ studied the conventional wire rope vibration isolator under three types of loading.

The dynamic response model of an O-type wire-cable vibration isolator is a very important tool for designing systems using the isolator as well as evaluating the responses of systems with such isolators. To date, no accurate physical model for the dynamic behavior of the isolator has been analytically derived. In most engineering applications, the behavior of the isolator is described by empirical or phenomenological models. The Bouc-Wen model [11, 12] and its normalized version [13] have been widely used to model many engineering structural systems [14] and describe various kinds of hysteretic systems such as magnetorheological dampers $[15,16]$. However, the original Bouc-Wen model cannot model the dynamic behavior of O-type wire-cable vibration isolators in tension-compression mode accurately for it can only describe symmetrical hysteresis loops. $\mathrm{Ni}$ et al. [5] proposed two modified Bouc-Wen models which are capable of representing the symmetric soft-hardening hysteresis loops and the asymmetric hysteresis loops with a hardening overlapping envelope, respectively. The parameters of Ni's model are identified using Ni's frequency domain identification method [17]. With Ni's method, to search for the optimal parameters, a set of initial values must be manually chosen. However, it is rather difficult to make a guess of a reasonable set of initial values in practice and therefore the method is often found failing to converge to a reasonable solution. Ikhouane and Gomis-Bellmunt proposed a limit cycle identification method [18]. This method does not rely on iteration algorithms and therefore is free of convergence problems. Making use of the limit cycle, Zhao et al. [19] proposed a two-stage method for identifying the parameters of a modified Bouc-Wen model that is capable of describing the dynamic behavior of the asymmetric hysteresis loops with hardening overlapping behaviors.

In the present work, periodic loading tests were conducted on eight types of the new O-type wire-cable vibration isolators to investigate the impact of the loading frequency, loading amplitude, and the isolator geometrics on

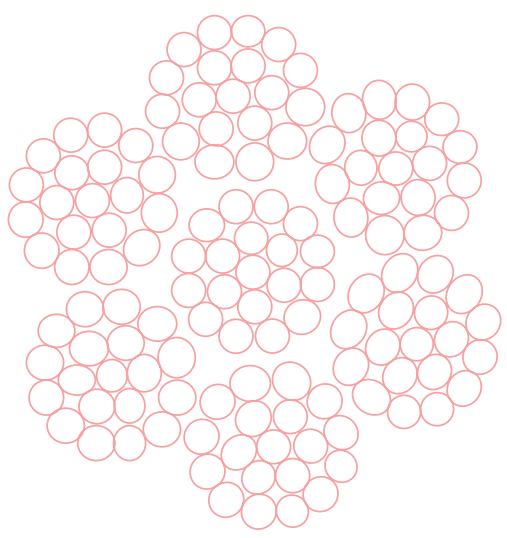

Figure 2: The cross section of the wire rope.

the dynamic characteristics of O-type wire rope vibration isolator. The isolators are tested under shear, roll, and tensioncompression loadings. A modified Bouc-Wen model is adopted to model the dynamic behavior of the O-type wirecable vibration isolators and the model parameters are identified using the experimental data. Finally, the steady state dynamic responses of an O-type wire-cable isolation-mass system in three modes are evaluated numerically.

\section{Periodic Loading Experiment}

Deformation controlled periodic loading experiments were carried out on several O-type wire-cable vibration isolators. The isolators are made of $6 \times 19$ IWSC wire ropes. The wire ropes are clustered in six strands of 19 steel wires each, which are wrapped around a central strand with 19 steel wires. The cross section of the wire rope is shown in Figure 2. The diameter of the wires that made up the wire rope is $0.4 \mathrm{~mm}$. The material of the wires is American Iron and Steel Institute (AISI) Stainless Steel Type 316.

2.1. Experiment Scheme. The isolator geometric parameters include the wire rope loop mean diameter $D$, the wire rope diameter $d$, wire number of rope loop $g$, horizontal angle of wire rope loop $\alpha$, loading amplitude $A$, and loading frequency $f$. To find out the loading amplitude and the isolator geometrics' impact on the dynamic response characteristics of the isolator, the tests were conducted following the scheme listed in Table 1.

The symbols $s, r$, and $t-c$ in the last column of Table 1 represent the shear, roll, and tension-compression loading, respectively.

2.2. Setup of the Experiment. The experiments were carried out on an elastomer test system, as shown in Figure 3. The isolators were fixed by the upper and lower clamps of the mechanical testing and simulation machine (MTS). The upper fixture of MTS vibrates periodically while the lower one is fixed throughout the experiment. In tests where the isolators are under shear or roll loading, two isolators are bolted together to avoid unbalanced load. The deformation 
TABLE 1: Experiment scheme.

\begin{tabular}{|c|c|c|c|c|c|c|c|c|}
\hline Isolator number & $D(\mathrm{~mm})$ & $d(\mathrm{~mm})$ & $D / d$ & $g$ & $\alpha\left(^{\circ}\right)$ & $A(\mathrm{~mm})$ & $f(\mathrm{~Hz})$ & Loading \\
\hline 1 & 63 & 4 & 15.75 & 8 & 60 & 3,5 & 1,5 & $s, r$, and $t-c$ \\
\hline 2 & 50 & 5 & 10 & 8 & 60 & 3,5 & 1,5 & $s, r$, and $t-c$ \\
\hline 3 & 55 & 5 & 11 & 8 & 60 & 3,5 & 1,5 & $s, r$, and $t-c$ \\
\hline 4 & 63 & 5 & 12.6 & 8 & 60 & $1-10$ & 1,5 & $s, r$, and $t-c$ \\
\hline 5 & 65 & 5 & 13 & 8 & 60 & 3,5 & 1,5 & $s, r$, and $t-c$ \\
\hline 6 & 63 & 6 & 105 & 8 & 60 & 1,2 & $1-19$ & $s, r$, and $t-c$ \\
\hline & & & & & & 3,5 & 1,5 & $s, r$, and $t-c$ \\
\hline 7 & 63 & 8 & 7.875 & $\begin{array}{c}8 \\
2,4,6\end{array}$ & 60 & 3,5 & 1,5 & $s, r$, and $t-c$ \\
\hline 8 & 63 & 6 & 10.5 & 2 & $90,80.87,71.49,61.56,50.59$ & 3,5 & 1,5 & $t-c$ \\
\hline
\end{tabular}

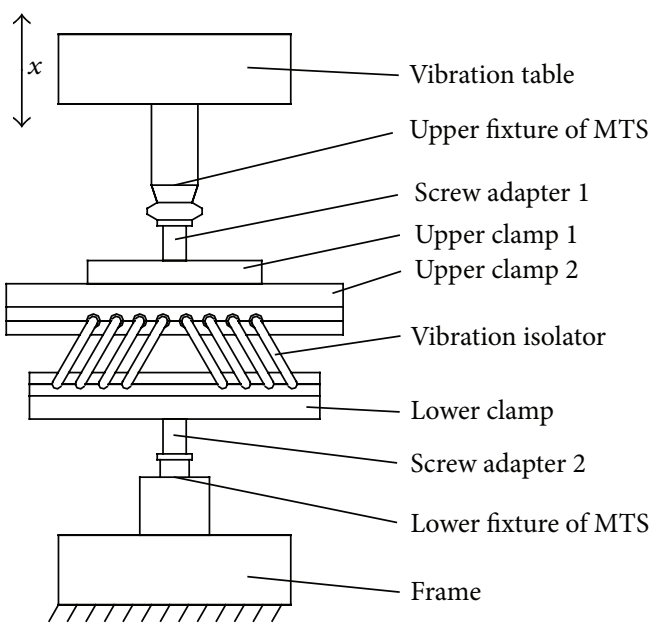

(a) Schematic diagram of the setup of an isolator under tension-compression loading

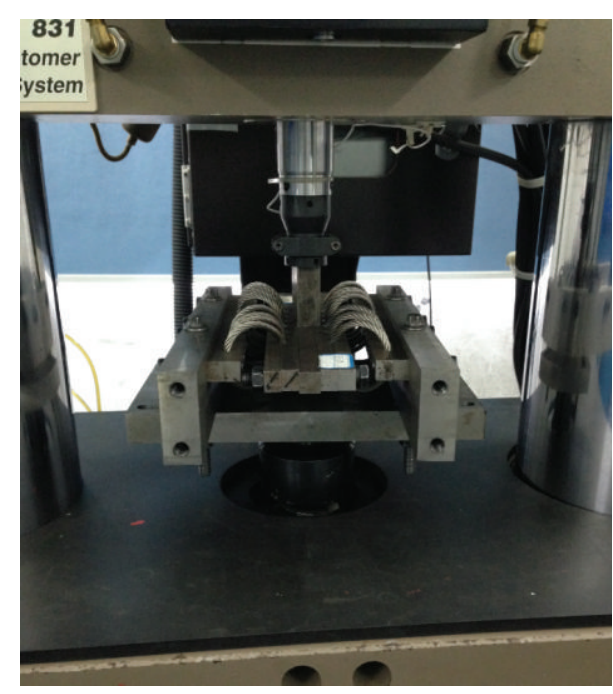

(c) The actual setup of an isolator under roll loading

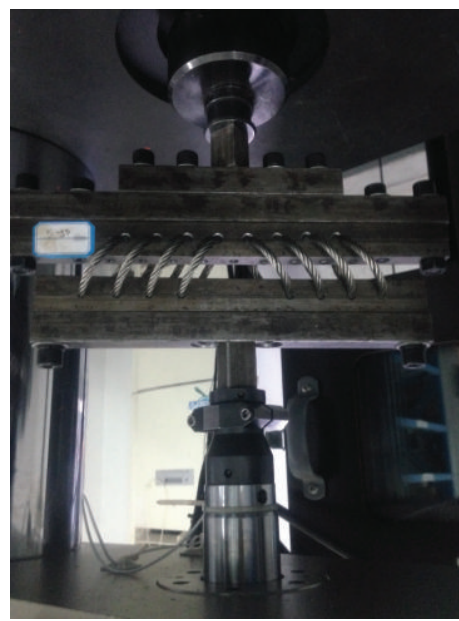

(b) The actual setup of an isolator under tension-compression loading

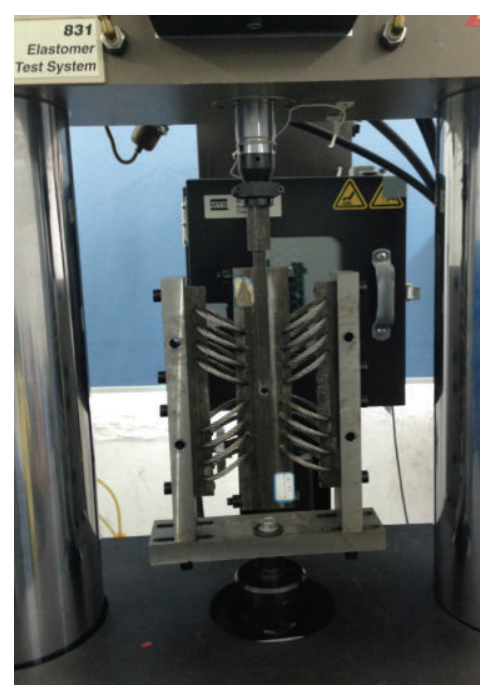

(d) The actual setup of an isolator under shear loading

FIGURE 3: Setup of the experiment. 


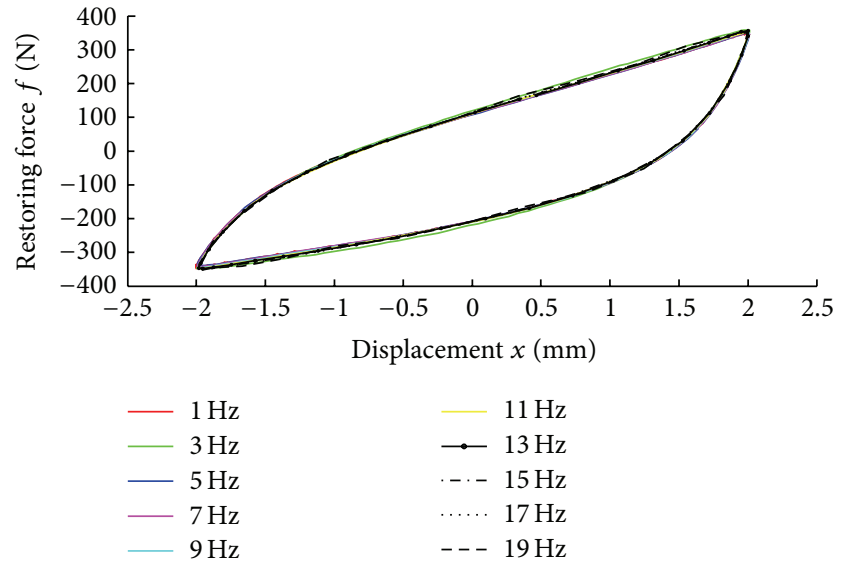

(a) Tension-compression loading

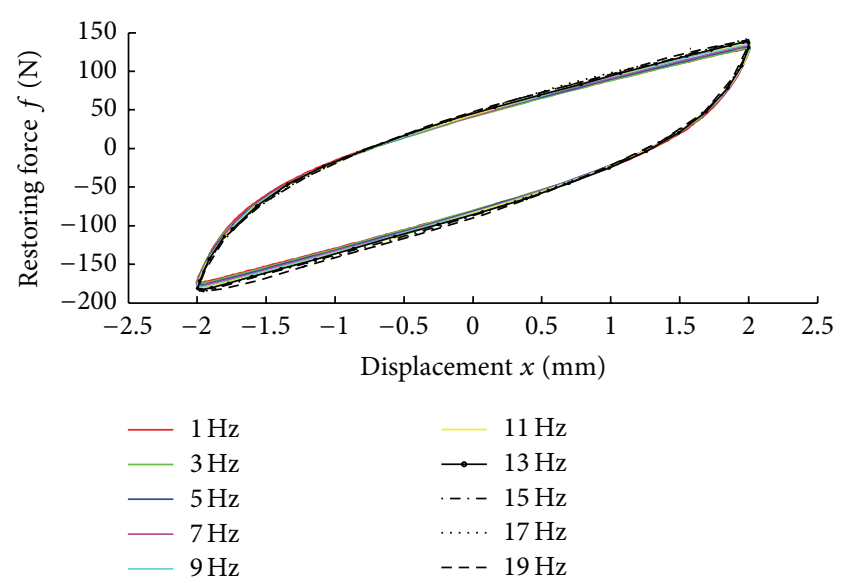

(b) Shear loading

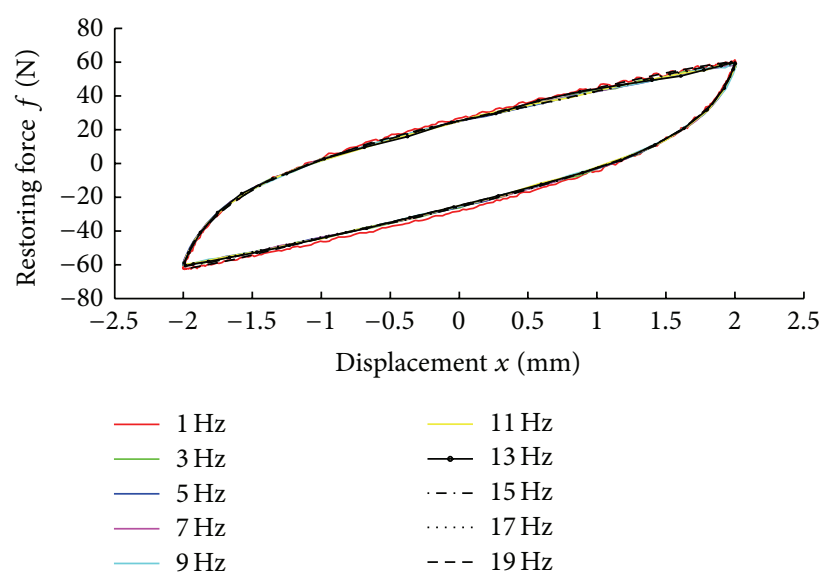

(c) Roll loading

FIGURE 4: Experimental hysteresis loops with different frequencies.

of an isolator is equivalent to the displacement of the upper clamp of MTS. The lower clamp of MTS is installed on top of a load cell. The displacement of the upper fixture of MTS is measured by a potentiometric linear displacement sensor. The restoring force and displacement signals are sampled synchronously. The sampling rate was chosen so as to collect 512 points per cycle according to the loading frequency. The sampling time was chosen to cover at least 15 loading cycles.

\subsection{Results}

2.3.1. The Dependence of Dynamic Characteristics on Excitation Frequency. Figure 4 shows the measured hysteresis loops for isolator number 6 under shear, roll, and tensioncompression loading, respectively. The loading frequency is set to be ranging from 1 to $19 \mathrm{~Hz}$. The loading amplitude is $A=2 \mathrm{~mm}$. It can be observed that the response of the O-type isolator is rate-independent in the tested frequency range. Such rate-independent nature is similar to conventional wirecable isolators $[2,8,10]$.
2.3.2. The Dependence of Dynamic Characteristics on Excitation Amplitude. Figure 5 shows the measured hysteresis loops for isolator number 4 in shear, roll, and tensioncompression loading, respectively. The loading amplitude is set to be ranging from 1 to $10 \mathrm{~mm}$ at the loading frequency $f=5 \mathrm{~Hz}$.

Figure 5(a) shows that the isolator exhibits significant asymmetric hysteresis loops under tension-compression loading and an envelope that contains all the hysteresis loops is presented by the loop with the largest loading amplitude. As the loading amplitude increases, stiffening and softening behaviors are observed on tension and compression sides, respectively. The asymmetry increases with the loading amplitude. The hysteresis loops under the shear and roll loading are similar as shown in Figures 5(b) and 5(c). The tested isolator generates symmetric hysteresis loops under both loadings owing to its symmetric configuration in these loading directions. For specific loading amplitude, the restoring force under the tension-compression loading is much greater than those under shear or the roll loadings. 


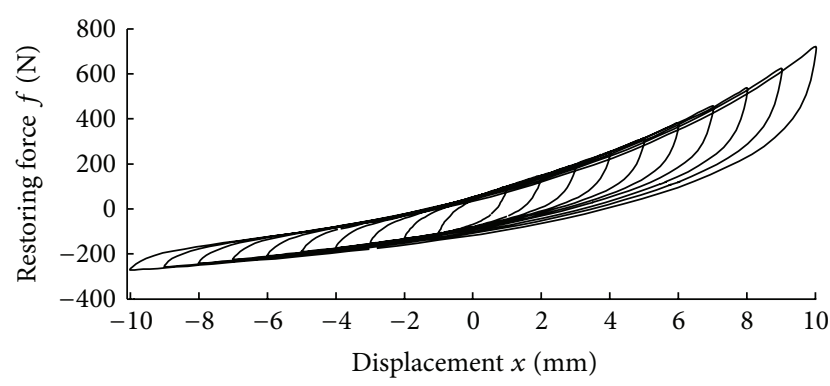

(a) Tension-compression loading

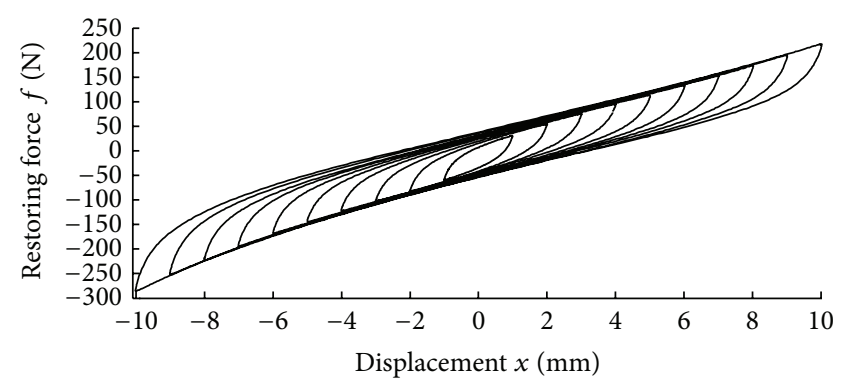

(b) Shear loading

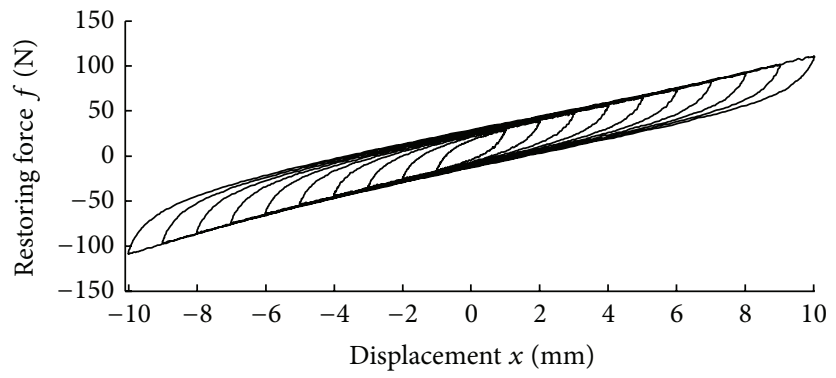

(c) Roll loading

FIGURE 5: Experimental hysteresis loops with different displacement amplitudes.

2.3.3. The Dependence of Dynamic Characteristics on Wire Rope Diameter. All the geometric parameters of isolators of number 1 , number 4 , number 6 , and number 7 are equivalent except the wire rope diameter. The measured hysteresis loops of the four isolators under shear, roll, and tensioncompression loading are plotted in Figure 6. The loading amplitude is set to be $A=5 \mathrm{~mm}$ at the frequency $f=5 \mathrm{~Hz}$.

It is obvious that stiffness and the dissipated energy of the isolator increase with the wire rope diameter. The variation of a relatively small $d$ can lead to a significant change of the stiffness. In other words, enlarging $d$ will have a stiffening effect on the isolator, and the stiffening effect tends to get strengthened as $d$ increases. However, according to Figure 6, if $d$ is small enough, the differences of the stiffness and the dissipated energy tend to get smaller as $d$ decreases. This implies that the effective stiffness is close to being linear when $d$ is small. Hence, the asymmetry is increasingly apparent in the tension-compression mode as the wire rope diameter $d$ increases.

2.3.4. The Dependence of Dynamic Characteristics on Wire Rope Loop Diameter. All the geometric parameters of isolators of number 2, number 3, number 4 , and number 5 are equivalent except the wire rope loop diameter. The measured hysteresis loops of the four isolators under shear, roll, and tension-compression loading are plotted in Figure 7. The loading amplitude is set to be $A=5 \mathrm{~mm}$ at the frequency $f=5 \mathrm{~Hz}$.

It is observed that stiffness tends to decrease as $D$ increases. The differences of the dissipated energy and the asymmetry of the hysteresis loops between the isolators are insignificant when the isolators are under tensioncompression loading. However, the dissipated energy increases with $D$ significantly when the isolators are under shear or roll loading.

2.3.5. The Dependence of Dynamic Characteristics on the Number and Horizontal Angle of the Wire Rope Loops. By changing the number of the wire rope loops in isolator number 7 , the relation between dependence of dynamic characteristics on the number of the wire rope loops is studied. The measured hysteresis loops are under tensioncompression loading. The loading amplitude is set to be $A=$ $5 \mathrm{~mm}$ at the frequency $f=5 \mathrm{~Hz}$. The measured hysteresis loops are plotted in Figure 8. Isolator number 8 is rigged with different horizontal wire rope angles so that the dependence of dynamic characteristics on the horizontal wire rope angle can be studied. The experimentally acquired hysteresis loops of isolator number 8 under tension-compression loading with loading amplitude $A=5 \mathrm{~mm}$ at the frequency $f=5 \mathrm{~Hz}$ are plotted in Figure 9.

It can be observed in Figure 8 that both the stiffness and dissipated energy tend to increase with the wire rope loop number. According to Figure 9, the stiffness tends to increase slightly as with $\alpha$.

2.3.6. The Dependence of Energy Dissipation on $A$ and Isolator Geometrics. The dissipated energy of the isolator is the area enclosed by the hysteresis loop. Figure 10 illustrates the hysteresis loop area varying with the loading amplitude $A$ ranging from 1 to $10 \mathrm{~mm}$ at the frequency $f=5 \mathrm{~Hz}$. Figure 11 illustrates the hysteresis loop area varying with different $D / d$ ratio with loading amplitude $A=5 \mathrm{~mm}$ and loading 


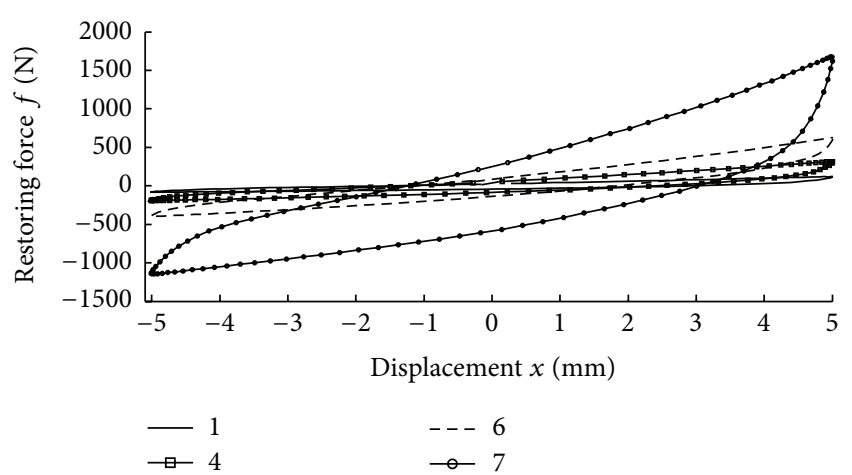

(a) Tension-compression loading

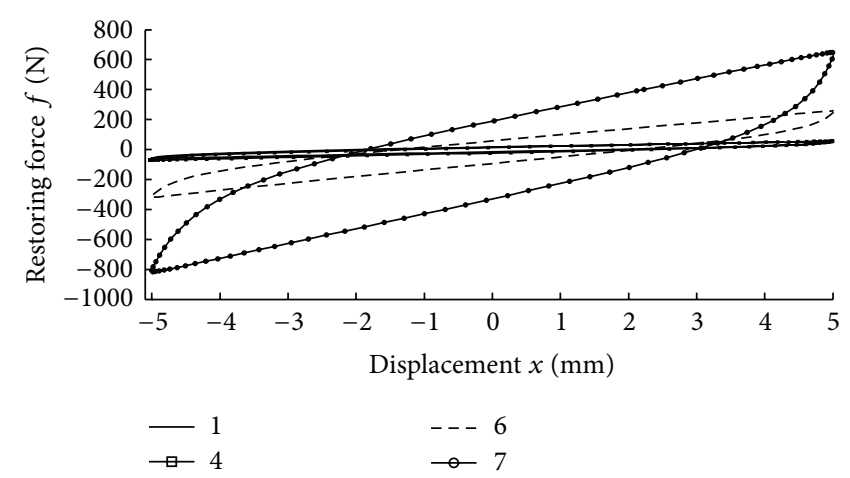

(b) Shear loading
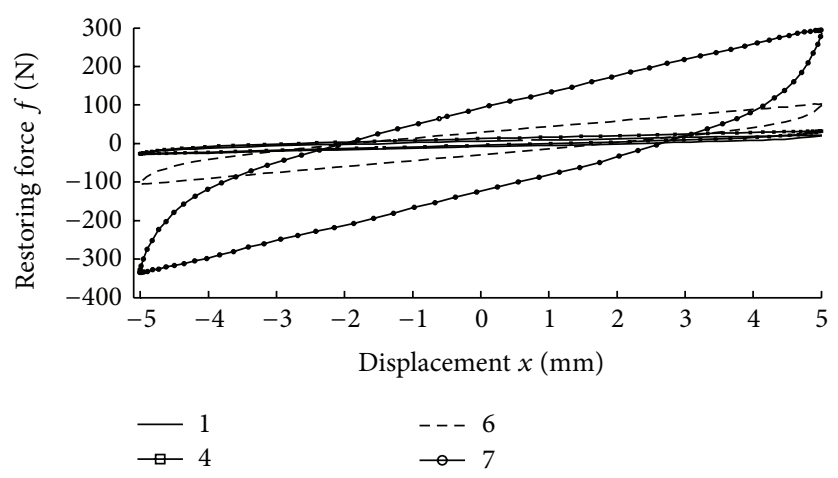

(c) Roll loading

FIGURE 6: Experimental hysteresis loops of isolators with different wire rope diameters.

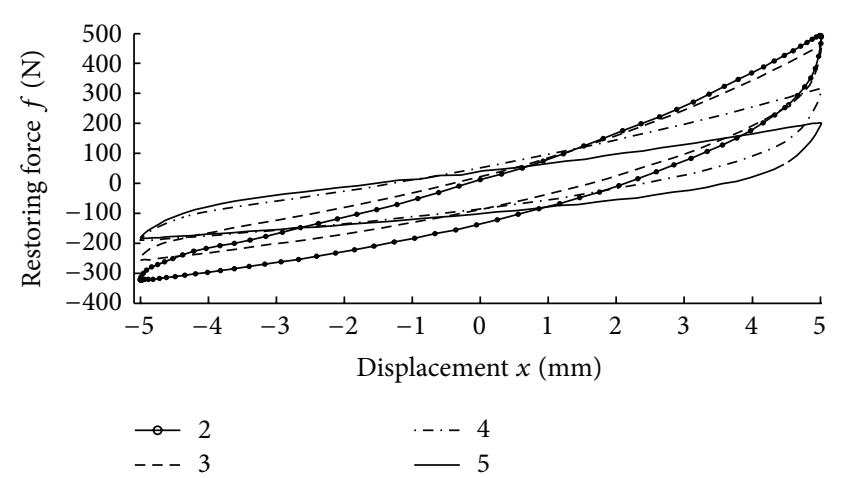

(a) Tension-compression loading

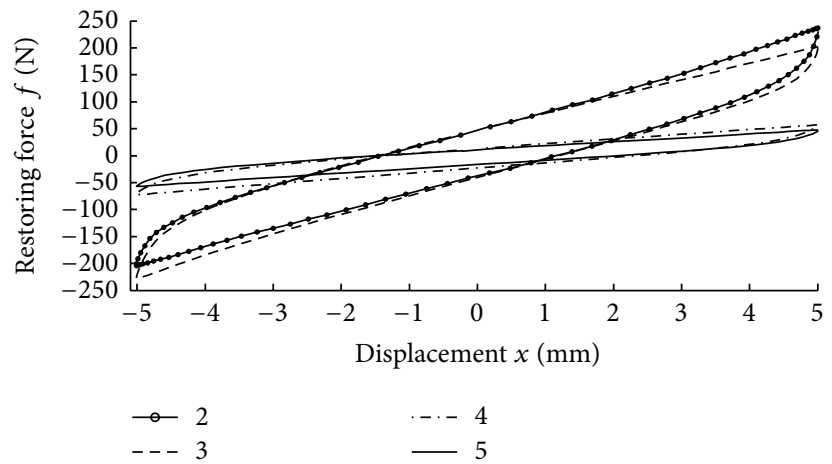

(b) Shear loading

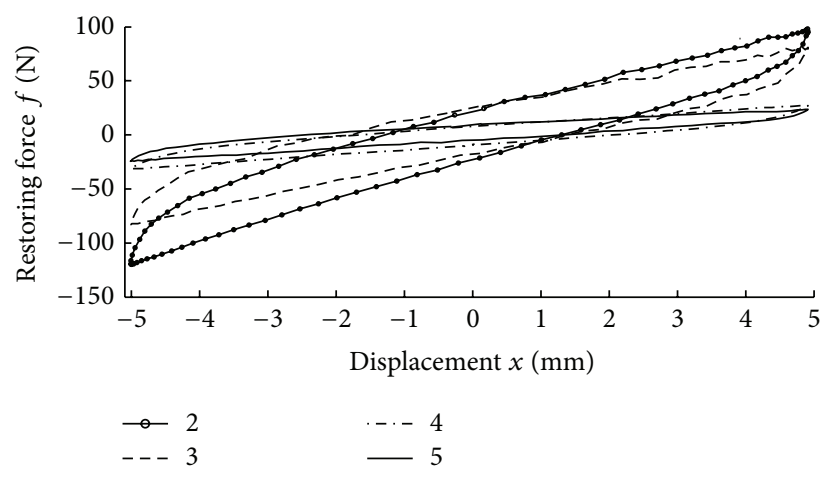

(c) Roll loading

FIGURE 7: Experimental hysteresis loops of isolators with different wire rope loop diameters. 


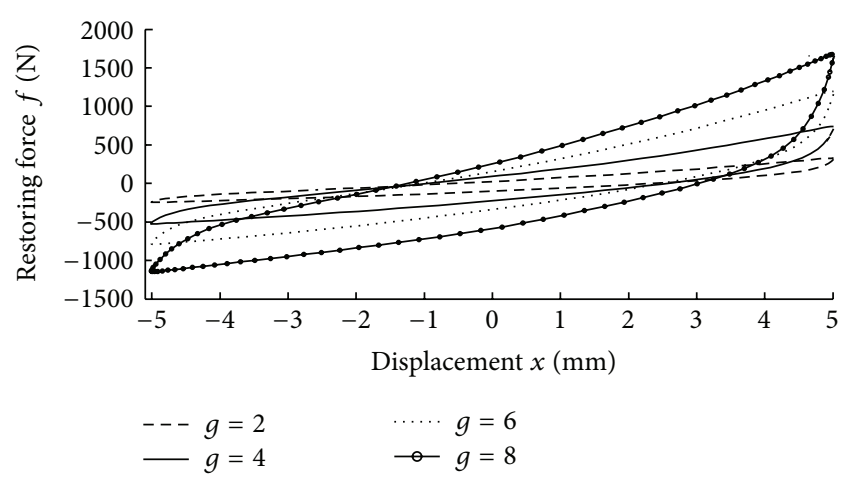

FIGURE 8: Measured hysteresis loops with different wire rope loop numbers.

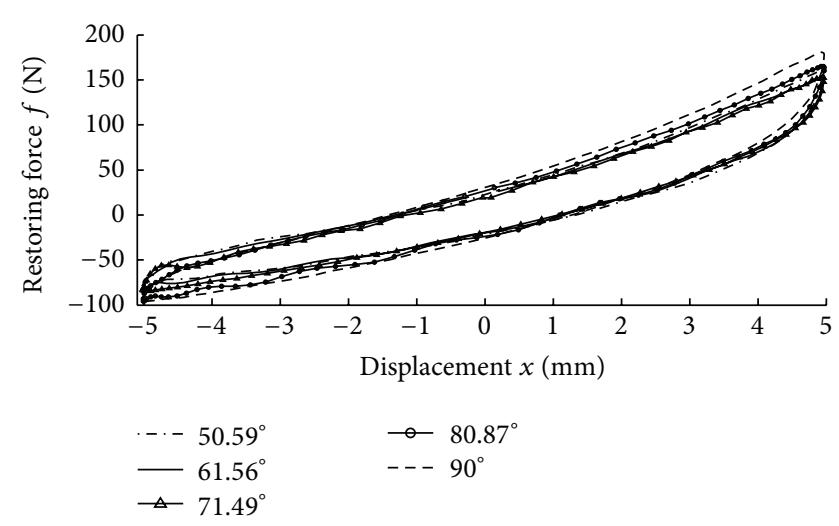

FIGURE 9: Measured hysteresis loops with different horizontal angle of wire rope loop.

frequency $f=5 \mathrm{~Hz}$. Figure 12 illustrates the hysteresis loop area varying with the number of wire rope loops with loading amplitude $A=5 \mathrm{~mm}$ and loading frequency $f=5 \mathrm{~Hz}$. Figure 13 illustrates the hysteresis loop area varying with horizontal angle of wire rope loops with loading amplitude $A=5 \mathrm{~mm}$ and loading frequency $f=5 \mathrm{~Hz}$.

It can be observed that the hysteresis loop area increases with the loading amplitude. The area-amplitude curve exhibits weak nonlinearity. The isolators offer much larger energy dissipation when loaded under the tensioncompression mode. It can be observed in Figure 11 that the relation between the hysteresis loop area and the $D / d$ ratio is not monotonic.

The hysteresis loop area increases nearly linearly with the number of the wire rope loops $g$. Figure 13 suggests that the hysteresis loop area decreases as the horizontal angle of wire rope loops $\alpha$ increases if $\alpha$ is small. When $\alpha$ reaches a certain value, the hysteresis loop area then begins to increase with $\alpha$. This is because the installation of the isolator is changed from the tension-compression mode to the combination of shear and tension-compression mode as the horizontal angle of wire rope loop $\alpha$ decreases.

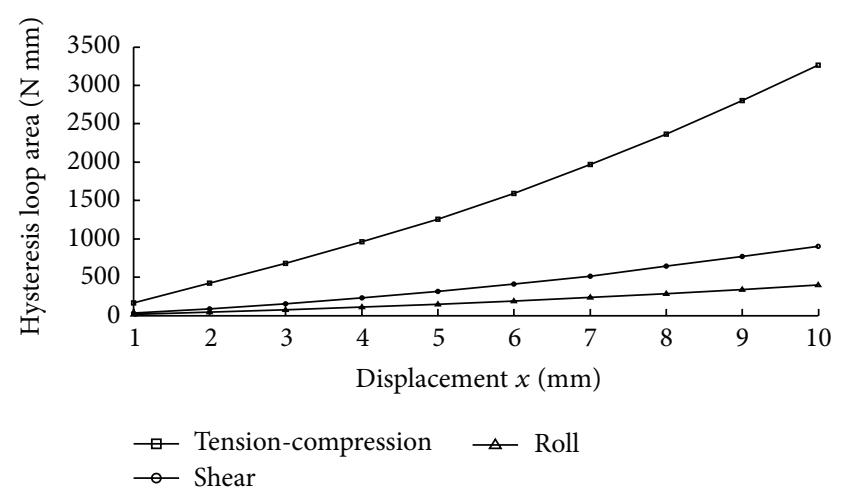

Figure 10: Hysteresis loop area versus loading amplitude.

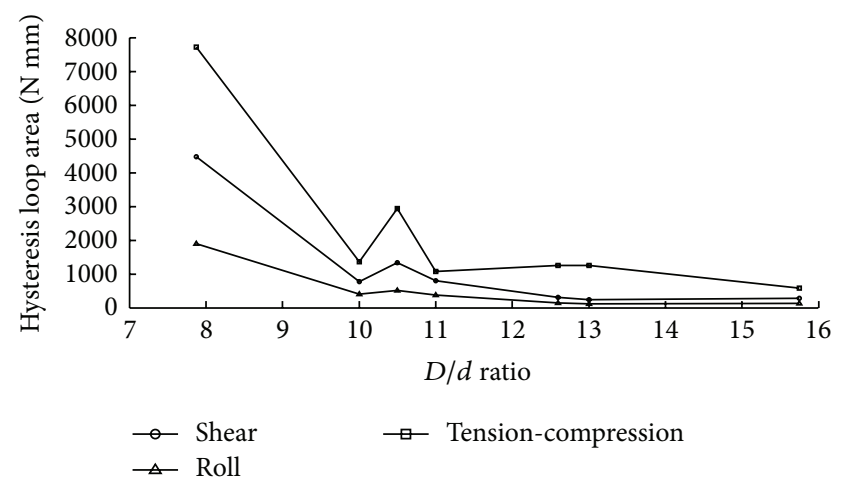

Figure 11: Hysteresis loop area versus $D / d$ ratio.

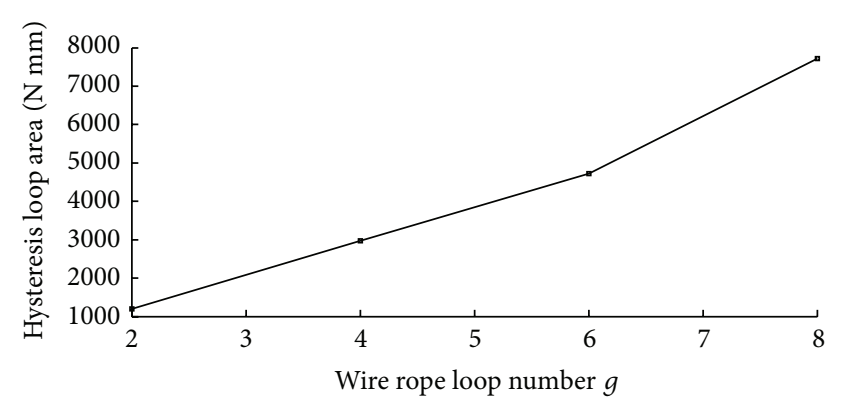

FIGURE 12: Hysteresis loop area versus the number of the wire rope loops.

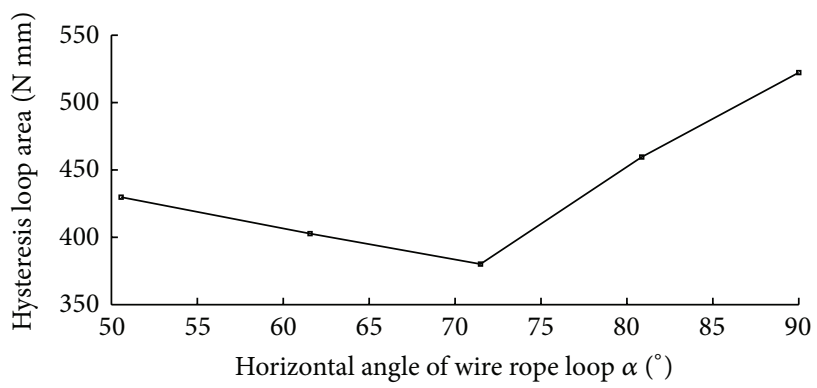

FIGURE 13: Hysteresis loop area versus the horizontal angle of the wire rope loops. 


\section{Modelling and Identification of the O-Type Wire-Cable Vibration Isolator}

3.1. Dynamic Response Model of the Isolator. The dynamic response model of the isolator is a rather important tool for designing dynamic systems with the isolator. As is observed in the aforementioned experiment, the isolator can generate both symmetric and asymmetric hysteresis loops depending on the way it is loaded. Such dynamic behavior can be readily described by Zhao et al's modified Bouc-Wen model [19] with a slight twist. The model is expressed as

$$
\begin{gathered}
\Phi(t)=F_{e}(x(t))+k_{z} z(t) F_{n}(x(t)), \\
F_{e}(x(t))=\sum_{i=0}^{N} k_{e i} x^{i}, \\
F_{n}(x(t))=\sum_{j=0}^{M} k_{n j} x^{j},
\end{gathered}
$$

where $t$ is the time, $x$ is the deformation of the isolator, $\Phi$ is the overall restoring force, $F_{e}, F_{n}$, and $z$ are the nonlinear elastic component, the nonlinear amplifier component, and the pure hysteretic component of the restoring force, respectively, $N$ and $M$ are the degrees of the polynomials, and $k_{e i}$ and $k_{n j}$ are the model parameters.

The pure hysteretic component $z$ is given by the normalized Bouc-Wen model $[13,19]$ as

$$
\dot{z}=\rho \dot{x}\left\{1-[\sigma \operatorname{sgn}(\dot{x}) \operatorname{sgn}(z)-\sigma+1]|z|^{n}\right\},
$$

where the over dot denotes the time derivation and $\rho, \sigma$, and $n$ are the model parameters.

Owing to the fact that $k_{z}$ is actually a redundant variable which can be combined with the coefficients of $F_{n}$ to simplify the calculation procedure, (1) can be recast as

$$
\Phi(t)=F_{e}(x(t))+F_{n}(x(t)) z(t) .
$$

3.2. Identification of the Model Parameters. The nonhysteretic parameters of the nonlinear amplifier $F_{n}$ and the nonlinear elastic component $F_{e}$ are identified first. Using the identification results, the hysteretic parameters are then identified.

3.2.1. Identification of Nonhysteretic Parameters. In order to achieve the parameter identification, a "bounded region" must be selected from a measured hysteresis loop. In the "bounded region," the hysteretic component of the response model can be simplified as a constant. Usually, the "bounded region" is the middle part of the measured hysteresis loop, as is illustrated in Figure 14.

The response of the pure hysteretic component $|z|$ is bounded to 1 when $x$ is in the "bounded region" [19]. In such case, the restoring force in the section can be derived as

$$
\begin{array}{ll}
\Phi_{l}=F_{e}+F_{n}, & \dot{x}>0, \\
\Phi_{u}=F_{e}-F_{n}, & \dot{x}<0,
\end{array}
$$

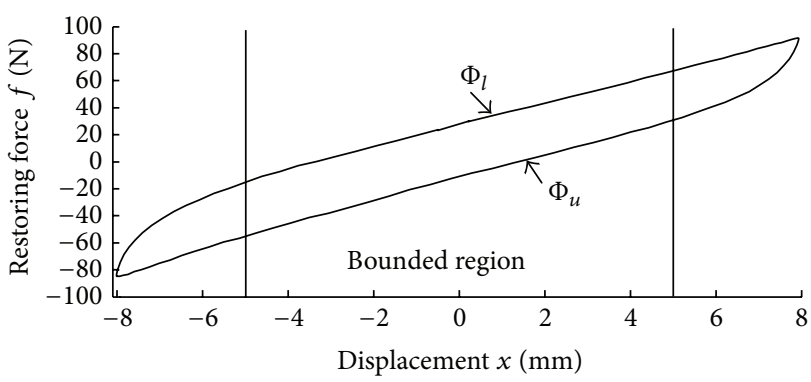

FIGURE 14: The selected "bounded region" of a measured hysteresis loop.

where $\Phi_{l}$ and $\Phi_{u}$ are the loading branch and the unloading branch of the hysteresis loop, respectively. $F_{n}$ and $F_{e}$ can be derived as

$$
\begin{aligned}
& F_{n}=\frac{\Phi_{l}-\Phi_{u}}{2}, \\
& F_{e}=\frac{\Phi_{l}+\Phi_{u}}{2} .
\end{aligned}
$$

The experimental data is a series of discrete data points; therefore, (7) should be recast in the discrete form

$$
F_{n k}=\frac{\Phi_{l k}-\Phi_{u k}}{2}
$$

where $k=1,2, \ldots, K$ denotes the $k$ th point of the discrete experimental data and $K$ is the length of the experimental data. Using (3) and (8), the following relation can be found:

$$
\mathbf{F}_{n}=\mathbf{k}_{n} \mathbf{y}_{M},
$$

where $\mathbf{k}_{n}=\left[k_{n 1}, k_{n 2}, \ldots, k_{n M}\right]$ is the nonlinear amplification coefficients vector, $\mathbf{F}_{n}=\left[F_{n 1}, F_{n 2}, \ldots, F_{n K}\right]$ is the nonlinear amplification vector, and $\mathbf{y}_{M}$ is the linearized deformation matrix for the nonlinear amplifier component. The matrix is given by

$$
\mathbf{y}_{M}=\left(\begin{array}{cccc}
x_{1} & x_{2} & \cdots & x_{K} \\
x_{1}^{2} & x_{2}^{2} & \cdots & x_{K}^{2} \\
\vdots & \vdots & \ddots & \vdots \\
x_{1}^{M} & x_{2}^{M} & \cdots & x_{K}^{M}
\end{array}\right)
$$

To obtain the coefficient vectors $\mathbf{k}_{n}$, a linear optimization problem can be formulated as

$$
\min f\left(\mathbf{k}_{n}\right)=\left\|\mathbf{F}_{n}-\mathbf{k}_{n} \mathbf{y}_{M}\right\|
$$

The solution to the problem denoted by (11), that is, $\mathbf{k}_{n}$, can be readily solved using linear least square method [20]. The parameters of the nonlinear elastic component $F_{e}$ can be derived through a similar process. 
3.2.2. Identification of Hysteretic Parameters. Using the identification result of $\mathbf{k}_{e}$ and $\mathbf{k}_{n}$, the hysteretic component $z$ can be derived in the discrete form as

$$
z_{k}=\frac{\Phi_{k}-F_{e_{k}}}{F_{n k}}=\frac{\Phi_{k}-\mathbf{k}_{e} \mathbf{y}_{N k}}{\mathbf{k}_{n} \mathbf{y}_{M k}},
$$

where $\Phi_{k}$ is the $k$ th restoring force data point of the experimental hysteresis loop and $\mathbf{y}_{N k}$ and $\mathbf{y}_{M k}$ denote the $k$ th column of $\mathbf{y}_{N}$ and $\mathbf{y}_{M}$, respectively. The limit cycle approach $[17,18]$ is applied to identify the hysteretic parameters. Rewrite (4) in the following piecewise form:

$$
z^{\prime}= \begin{cases}\rho\left(1-z^{n}\right) & \dot{x}>0, z \geq 0, \\ \rho\left[1-(1-2 \sigma)(-z)^{n}\right] & \dot{x}>0, z<0, \\ \rho\left[1-(1-2 \sigma) z^{n}\right] & \dot{x}<0, z \geq 0, \\ \rho\left[1-(-z)^{n}\right] & \dot{x}<0, z<0,\end{cases}
$$

where the symbol $\left({ }^{\prime}\right)$ denotes the derivation with respect to $x$. Using (12) and (13), the hysteretic parameters $\rho, \sigma$, and $n$ can be easily identified using experiment data with a limit cycle identification method $[18,19]$.

At this point, all the model parameters are identified without any nonlinear iteration procedures; therefore, the identification is very fast and requires no guesses for any of the parameters.

3.2.3. Processing of the Experimental Data. In practice, the measured data will inevitably be contaminated by noises which could cause significant error when the data is used to identify the model parameters of the isolators. Hence, the measured data must be processed before the identification process. A simple yet effective way of denoising is the moving average method [21]. This method would be sufficient if the experimental data is not severely contaminated. For the case where the experimental data is severely contaminated, the data should be filtered using digital low pass filters before they are used in the identification process. When the sampling frequency satisfies some specific conditions [22], an effective frequency domain noncausal filtering method proposed by $\mathrm{Ni}$ et al. [17] could be adopted.

3.2.4. Identification Results. With the model and the identification method, the model parameters of isolator number 4 in shear, the roll, and tension-compression modes are identified and listed in Table 2. The hysteresis loops predicted using the identification results are plotted in Figure 15. It can be observed from Figure 15 that the predicted hysteresis coincides with the measured loops well.

\section{Evaluation of the Dynamic Response}

With the dynamic response model the response of systems with the isolator can be evaluated. Figure 16 illustrates the configuration of three systems consisting of mass blocks and O-type wire-cable vibration isolators. The isolators are installed in different ways so that they are subject to shear, roll, and tension-compression loading, respectively.
TABLE 2: Model parameter identification results.

\begin{tabular}{lccc}
\hline Model parameter & \multicolumn{3}{c}{ Mode } \\
\hline$k_{e i}$ & $\mathrm{~s}$ & $\mathrm{r}$ & $\mathrm{t}-\mathrm{c}$ \\
$k_{e 0}$ & -8.7845 & 7.7460 & -23.7263 \\
$k_{e 1}$ & 18.9590 & 8.4184 & 33.5017 \\
$k_{e 2}$ & -0.1800 & -0.0537 & 1.9960 \\
$k_{e 3}$ & 0 & 0 & 0.0722 \\
$k_{e 4}$ & 0 & 0 & 0.0023 \\
$k_{n j}$ & & & \\
$k_{n 0}$ & 42.0757 & 19.2852 & 71.19495 \\
$k_{n 1}$ & -0.15915 & -0.07229 & 6.91807 \\
$k_{n 2}$ & 0 & 0 & 0.2064 \\
$k_{n 3}$ & 0 & 0 & -0.05694 \\
Hysteretic parameters & & & \\
$\rho$ & 1.4503 & 1.1669 & 1.4095 \\
$\sigma$ & 2.2624 & 2.1586 & 2.8049 \\
$n$ & 0.8793 & 0.8998 & 0.8646 \\
\hline
\end{tabular}

Suppose that the system is subjected to the base excitation displacement $x_{g}(t)=A \sin (2 \pi f t)$ and the displacement of the mass block relative to the base or the deformation of the isolator is denoted by the symbol $x(t)$. The equation of motion of the system is expressed as

$$
m\left(\ddot{x}(t)+\ddot{x}_{g}(t)\right)=m g-\Phi(t) .
$$

When the system is in the static state, the following conditions are met:

$$
\begin{aligned}
& x(t)=x_{g}(t)=0, \\
& \dot{x}(t)=\dot{x}_{g}(t)=0, \\
& \ddot{x}(t)=\ddot{x}_{g}(t)=0 .
\end{aligned}
$$

In this case, by substituting (2)-(5) and (15) into (14), one can find that the gravity and the constant term of the nonlinear elastic component of the dynamic response model of the isolator are counteracted; namely,

$$
m g-k_{e 0}=0 .
$$

Using (2)-(5) and (16), the motion equation (14) can be rewritten as

$$
\begin{gathered}
m\left(\ddot{x}(t)+\ddot{x}_{g}(t)\right)+\sum_{i=1}^{N} k_{e i} x^{i}(t) \\
+z(t) \sum_{j=0}^{M} k_{n j} x^{j}(t)=0 .
\end{gathered}
$$

The Runge-Kutta method [23] is employed to solve (17) and study the frequency response of the systems numerically. The frequency response is obtained through a numerical frequency sweep process. In order to check if the system features multivalued responses, the excitation frequency is 


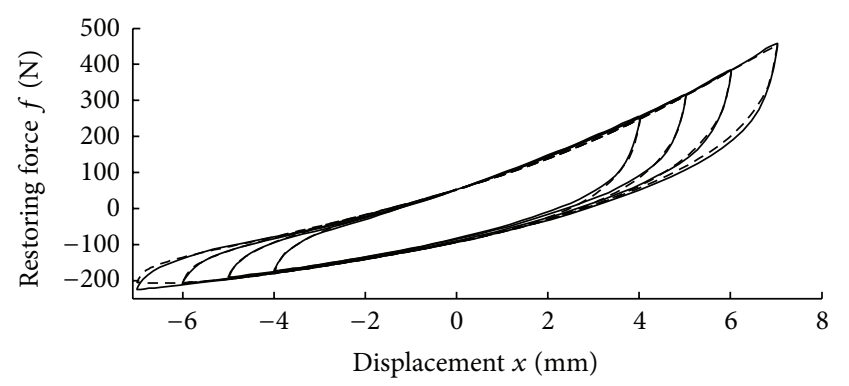

- Test - - - Predicted

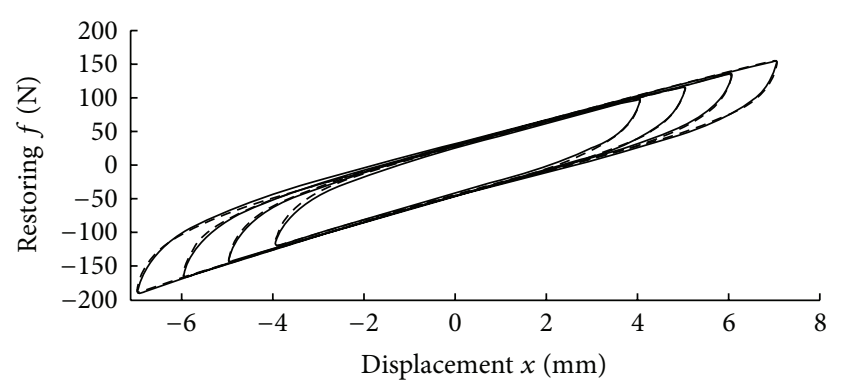

_ Test

- - - Predicted

(a) Tension-compression loading

(b) Shear loading

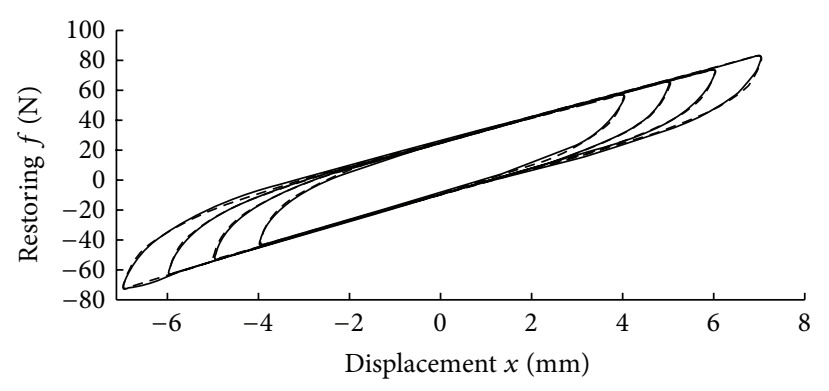

- Test

- - - Predicted

(c) Roll loading

FIGURE 15: The tested and predicted hysteresis loops.

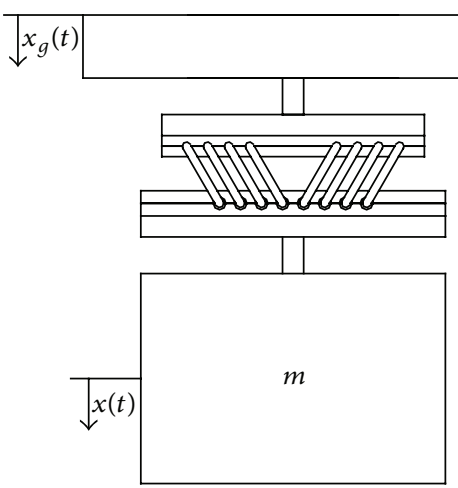

(a) Tension-compression loading

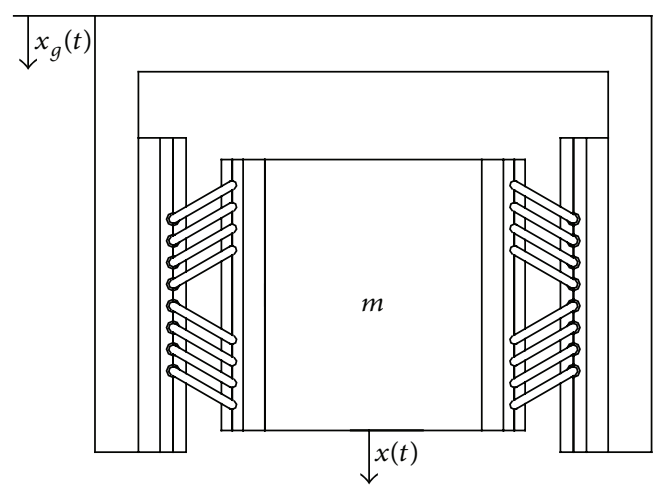

(b) Shear loading

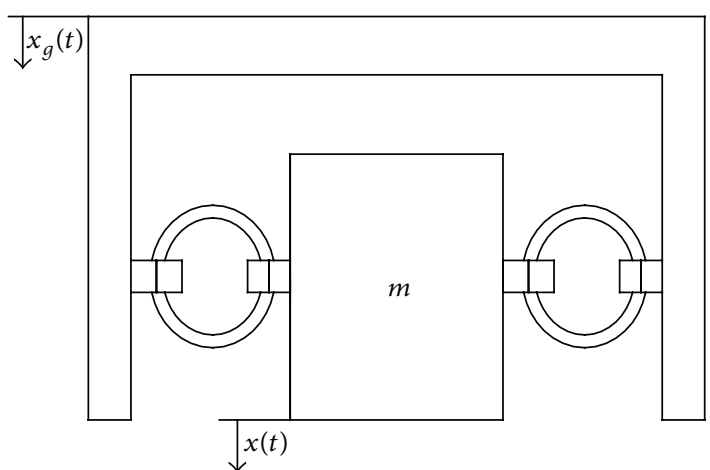

(c) Roll loading

FIGURE 16: Systems with the isolator. 


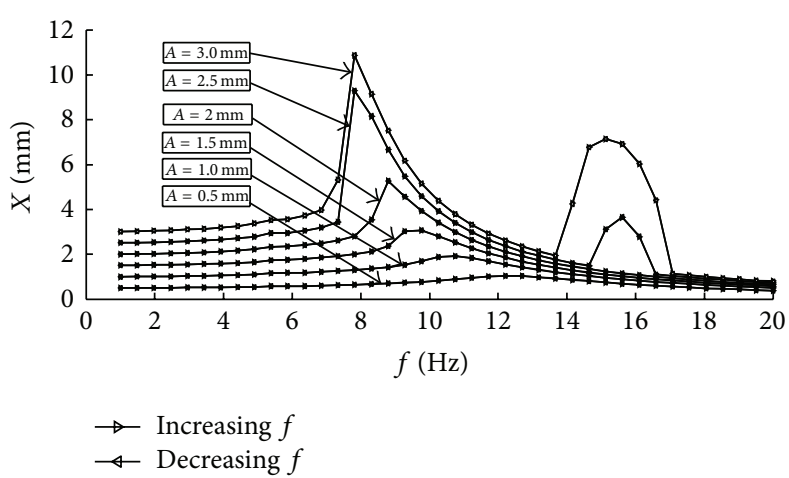

(a) Tension-compression loading

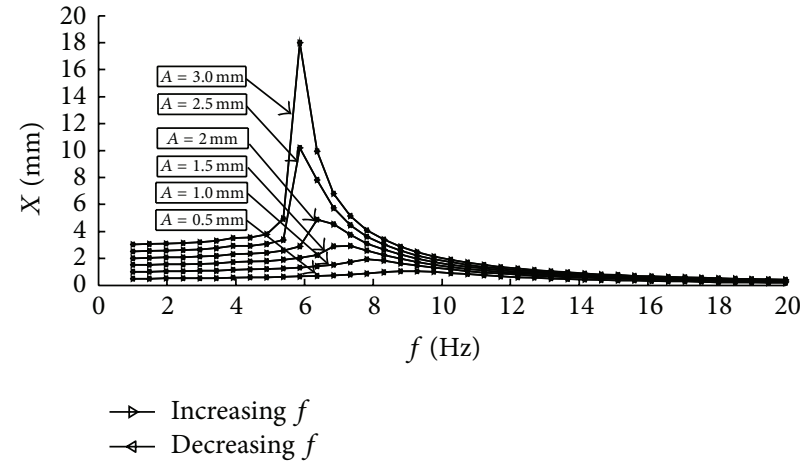

(b) Shear loading

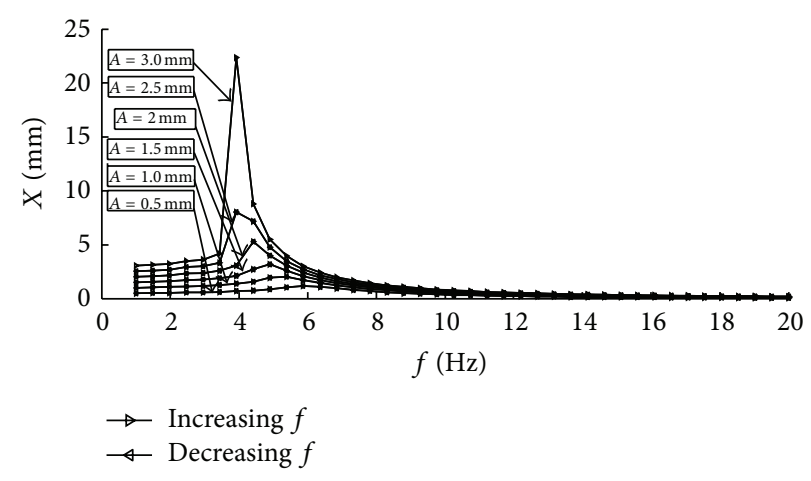

(c) Roll loading

FIGURE 17: The simulated frequency response curves of relative displacement.

firstly increased from the start frequency to the upper limit and then decreased to the start frequency when performing the sweep. The base displacement amplitude $A$ is chosen to be $0.5 \mathrm{~mm}, 1.0 \mathrm{~mm}, 1.5 \mathrm{~mm}, 2.0 \mathrm{~mm}, 2.5 \mathrm{~mm}$, and $3.0 \mathrm{~mm}$ in the current simulation. The range of the base displacement frequency $f$ is simulated as $1-20 \mathrm{~Hz}$. Let the amplitude of the displacement of the mass block relative to the ground be denoted by the symbol $X$. Figure 17 illustrates the computed frequency response curves of the system in the case $m=$ $15 \mathrm{~kg}$.

The frequency response curves show clearly the nonlinear feature of the wire-cable isolator system. Significant nonlinear behaviors such as evident second-order subharmonic resonance can be observed in the tension-compression mode while no subharmonic resonances exist in shear, roll modes. This is because, as aforementioned, the system features stronger nonlinearity when subject to tension-compression loading. Moreover, the resonant frequencies shift to the left as the excitation amplitude increases. This is similar to the behavior of convention wire-cable isolators [17]. Furthermore, with the same mass and the isolator, the primary resonant frequency of the system in the tension-compression mode is greater than that in the shear mode and is almost twice that in the roll mode. When the excitation amplitude is small, the system exhibits similar level of resonant amplitudes in all modes. However, when the excitation amplitude exceeds a certain level, the resonant amplitudes in the shear and roll modes are almost twice the amplitude in the tensioncompression mode. This indicates that the isolator is more effective in the tension-compression mode. No multivalued responses are found in the present system.

\section{Conclusion}

A series of dynamic tests were performed on several Otype wire-cable vibration isolators. The experimental results showed that the output of the isolator is almost independent of the loading frequency, however, strongly affected by the loading amplitude. As the loading amplitude increases, stiffening and softening behaviors are observed in tension and compression directions, respectively. The restoring forceloading hysteresis loops showed apparent asymmetry when the loading amplitude is large enough. However, the hysteresis loops are symmetric when the isolators were subject to shear and roll loadings. The energy dissipation capability of the isolators is strongest when it is under tensioncompression loading. The dependence of the stiffness and the energy dissipation capability of the isolators on the isolator geometrics was studied and reference was provided for the design and manufacturing of the isolator for engineering applications.

A phenomenological model was adopted to model the dynamic response of the isolator. The model parameters were identified using the experimental data. The identification process does not depend on nonlinear iterative algorithms and therefore is free from convergence problems. The hysteresis loops generated using the identification results agree with 
the measured hysteresis loops well, indicating the model and identification method are fit for the O-type isolator.

Based on the established dynamic response model of the isolator, the steady state dynamic response of a wirecable isolator-mass system was analyzed using RungeKutta method. The analysis results show that the frequency response characteristics of the system where the isolator is under tension-compression loading are significantly different from those under shear and roll loadings. For example, the resonance frequency is higher and the resonance amplitude is smaller. This is because the isolator features larger stiffness and better damping capability when it is the under tensioncompression loading. Significant nonlinear behaviors such as subharmonic resonance can be observed when the excitation amplitude is large enough and when the isolator is subject to tension-compression loading. However, no subharmonic resonance is found for isolators under shear or roll loadings. This suggests that the isolator features stronger nonlinearity in the tension-compression mode.

\section{Conflict of Interests}

The authors declare that there is no conflict of interests regarding the publication of this paper.

\section{Acknowledgments}

The study is supported by the National Basic Research Program of China (973 Program 2014CB049403), the National Science Foundation of China (51175525), and the State Key Laboratory Fund Project of Chongqing University (0301002109137).

\section{References}

[1] S. Chaudhuri and B. Kushwaha, "Wire rope based vibration isolation fixture for road transportation of heavy defence cargo," in Vibration Problems ICOVP-2007, Springer Proceedings in Physics, pp. 61-67, Springer, Berlin, Germany, 2008.

[2] G. F. Demetriades, M. C. Constantinou, and A. M. Reinhorn, "Study of wire rope systems for seismic protection of equipment in buildings," Engineering Structures, vol. 15, no. 5, pp. 321-334, 1993.

[3] G. Di Massa, S. Pagano, E. Rocca, and S. Strano, "Sensitive equipments on WRS-BTU isolators," Meccanica, vol. 48, no. 7, pp. 1777-1790, 2013.

[4] M. L. Tinker and M. A. Cutchins, "Damping phenomena in a wire rope vibration isolation system," Journal of Sound and Vibration, vol. 157, no. 1, pp. 7-18, 1992.

[5] Y. Q. Ni, J. M. Ko, C. W. Wong, and S. Zfaan, "Modelling and identification of a wire-cable viberation isolater via a cyclic loading test part 1: and experiment and model developement," Proceedings of the Institution of Mechanical Engineers I: Journal of Systems and Control Engineering, vol. 213, no. 3, pp. 163-171, 1999.

[6] S. Wang, Y. Zhao, J. Zhou, C. Li, and X. Li, "Static response of stranded wire helical springs to axial loads: a two-state model," Proceedings of the Institution of Mechanical Engineers, Part C: Journal of Mechanical Engineering Science, vol. 227, no. 7, pp. 1608-1618, 2013.
[7] X. Gong and Y. Tang, "New method for modeling of a nonlinear vibration system with hysteresis characteristics," Chinese Journal of Mechanical Engineering, vol. 35, no. 4, pp. 11-14, 1999.

[8] R. R. Gerges, "Model for the force-displacement relationship of wire rope springs," Journal of Aerospace Engineering, vol. 21, no. 1, pp. 1-9, 2008.

[9] R. R. Gerges and B. J. Vickery, "Design of tuned mass dampers incorporating wire rope springs: part I: dynamic representation of wire rope springs," Engineering Structures, vol. 27, no. 5, pp. 653-661, 2005.

[10] Y. Q. Ni, J. M. Ko, and C. W. Wong, "Identification of nonlinear hysteretic isolators from periodic vibration tests," Journal of Sound and Vibration, vol. 217, no. 4, pp. 737-756, 1998.

[11] R. Bouc, "Forced vibration of mechanical systems with hysteresis," in Proceedings of the 4th Conference on Nonlinear Oscillations, Prague, Czech Republic, 1967.

[12] Y. K. Wen, "Method for random vibration of hysteretic systems," Journal of the Engineering Mechanics Division, vol. 102, no. 2, pp. 249-263, 1976.

[13] F. Ikhouane and J. Rodellar, "On the hysteretic Bouc-Wen model. I. Forced limit cycle characterization," Nonlinear Dynamics, vol. 42, no. 1, pp. 63-78, 2005.

[14] Y. Zhang, "Stochastic responses of multi-degree-of-freedom uncertain hysteretic systems," Shock and Vibration, vol. 18, no. 1-2, pp. 387-396, 2011.

[15] W. Zhu and X. T. Rui, "Semiactive vibration control using a magnetorheological damper and a magnetorheological elastomer based on the Bouc-Wen model," Shock and Vibration, vol. 2014, Article ID 405421, 10 pages, 2014.

[16] A. M. Tusset, J. M. Balthazar, and J. L. Felix, "On elimination of chaotic behavior in a non-ideal portal frame structural system, using both passive and active controls," Journal of Vibration and Control, vol. 19, no. 6, pp. 803-813, 2013.

[17] Y. Q. Ni, J. M. Ko, C. W. Wong, and S. Zhan, "Modelling and identification of a wire-cable vibration isolator via a cyclic loading test Part 2: Identification and response prediction," Proceedings of the Institution of Mechanical Engineers I: Journal of Systems and Control Engineering, vol. 213, no. 3, pp. 173-182, 1999.

[18] F. Ikhouane and O. Gomis-Bellmunt, "A limit cycle approach for the parametric identification of hysteretic systems," Systems \& Control Letters, vol. 57, no. 8, pp. 663-669, 2008.

[19] Y. Zhao, S. L. Wang, J. Zhou, C. Li, and C. Cheng, "Modeling and identification of the dynamic behavior of stranded wire helical springs," Journal of Vibroengineering, vol. 15, no. 1, pp. 326-339, 2013.

[20] P. E. Gill, W. Murray, and M. H. Wright, Practical Optimization, Academic Press, London, UK, 1981.

[21] C. E. Armstrong, "Moving averages," The American Statistician, vol. 3 , no. $4,1949$.

[22] J. M. Ko, Y. Q. Ni, and Q. L. Tian, "Hysteretic behavior and empirical modeling of a wire-cable vibration isolator," The International Journal of Analytical and Experimental Modal Analysis, vol. 7, no. 2, pp. 111-127, 1992.

[23] H. Press, A. Teukolsky, T. Vetterling et al., Numerical Recipes in C++: The Art of Computer Programming, Cambridge University Press, New York, NY, USA, 2002. 

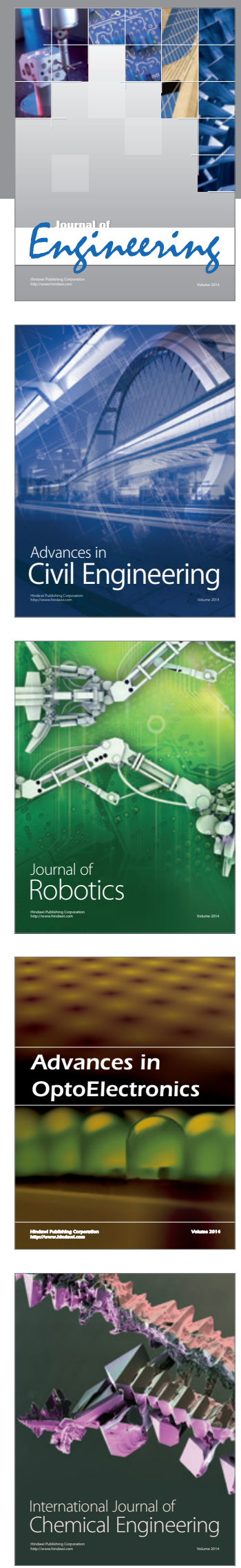

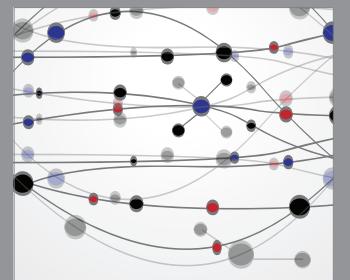

The Scientific World Journal
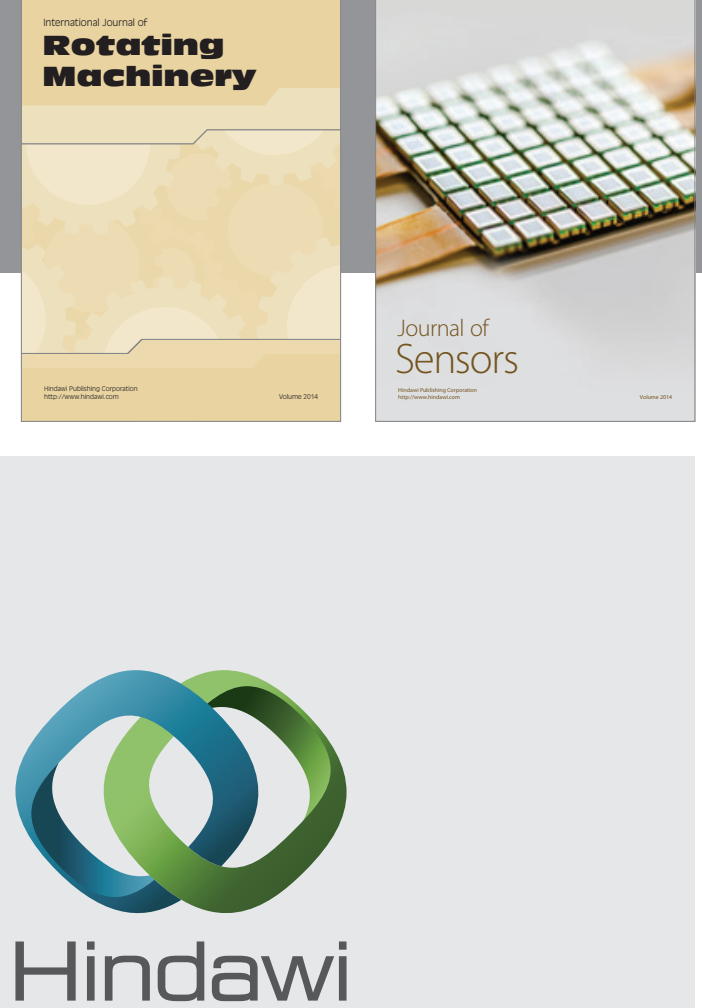

Submit your manuscripts at http://www.hindawi.com
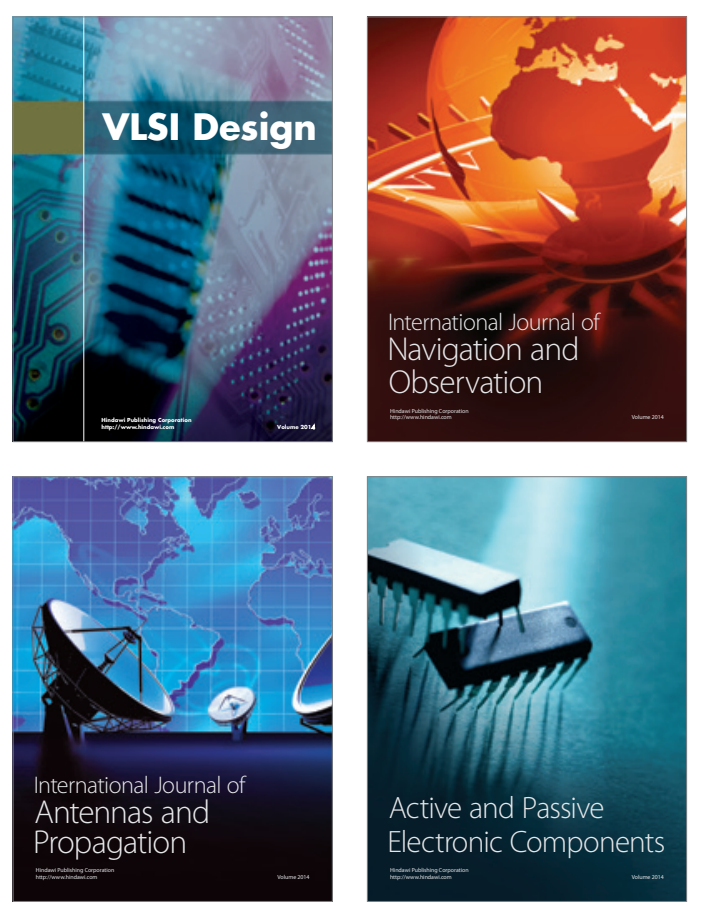
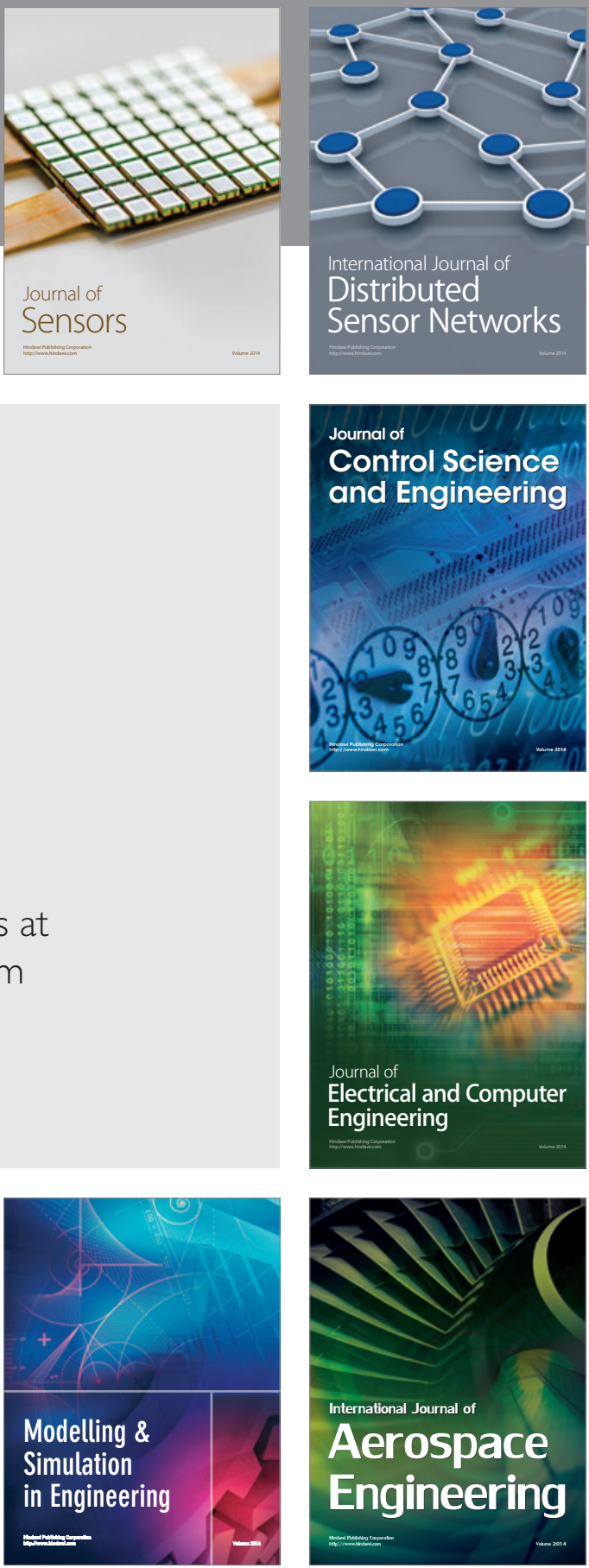

Journal of

Control Science

and Engineering
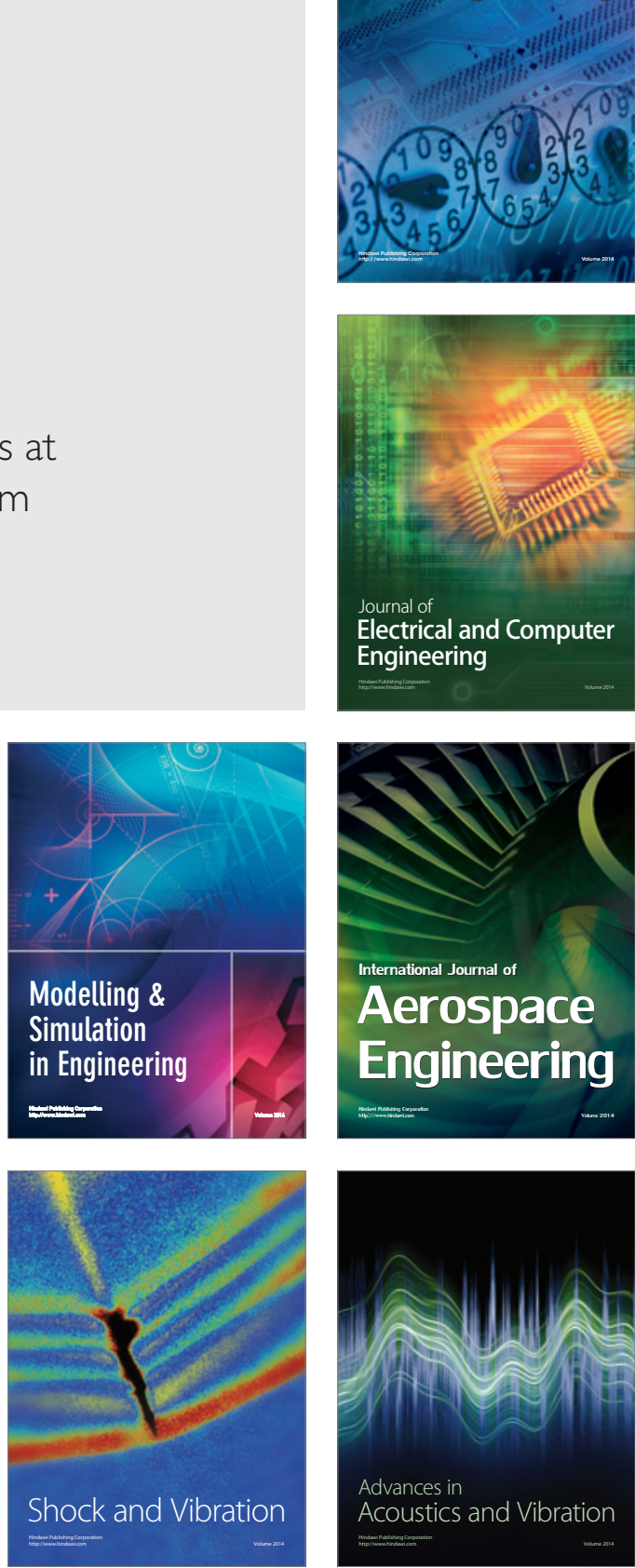